\title{
Usability evaluation of navigation tasks by people with intellectual disabilities: a Google and SAPO comparative study regarding different interaction modalities
}

\author{
Tânia Rocha ${ }^{1,2} \cdot$ Diana Carvalho ${ }^{1,2} \cdot$ Maximino Bessa $^{1,2} \cdot$ Sofia Reis $^{3}$. \\ Luís Magalhães ${ }^{4}$
}

Published online: 5 August 2016

(c) Springer-Verlag Berlin Heidelberg 2016

\begin{abstract}
This paper presents a case study regarding the usability evaluation of navigation tasks by people with intellectual disabilities. The aim was to investigate the factors affecting usability, by comparing their user-Web interactions and underline the difficulties observed. For that purpose, two distinct study phases were performed: the first consisted in comparing interaction using two different search engines' layouts (Google and SAPO) and the second phase consisted in a preliminary evaluation to analyze how users performed the tasks with the usual input devices (keyboard and mouse) and provide an alternative interface to help overcome possible interaction problems and enhance autonomy. For the latter, we compared two different interfaces: a WIMP-based one and speech-based one. The main results obtained showed that users had a better performance with Google (with a simpler layout) than with SAPO (with a complex layout), and despite displaying a good keyboard handling ability, they did not show autonomy using this input device (due to the need for reading/ writing when handling this device). In this perspective, Google's speech recognition application could indeed be considered an alternative for interaction. However, we found that the speech recognition interface is not as robust as it should be: it could be more precise and less prone to errors due to poor word pronunciation. After this two-
\end{abstract}

Tânia Rocha

trocha@utad.pt

1 University of Trás-os-MOntes e Alto Douro, Vila Real, Portugal

2 INESC TEC/UTAD, Vila Real, Portugal

3 University of Aveiro, Aveiro, Portugal

4 University of Minho, Braga, Portugal phased study, we think we may be able to infer some recommendations to be used by developers in order to create more intuitive layouts for easy navigation regarding this group of people, and thereby facilitate digital inclusion.

Keywords Web search · Web navigation tasks · Google · SAPO $\cdot$ Intellectual disability

\section{Introduction}

Today, the evolution of the information society justifies concerns about universal access to services provided online in order to improve people's quality of life. The existing gap between those who could keep up with and embrace the technological advances, and those that could not, represents an obstacle for all communities. This gap is considered a digital divide that reflects the deprivation of the access to ICTs to some part of the community [9].

The aim of Web accessibility is to guarantee that Web contents can be accessed and used by all potential users, regardless of their cognitive or motor limitations or the various contexts of use $[5,11]$. This concept is frequently discussed and analyzed nowadays, but there is a long way to go in order to make web content accessible for all, namely for people with intellectual disability.

Intellectual disability characterizes itself by the fact that an individual has an intellectual quotient significantly below average and by limitations in the performance of functioning capacities in daily life areas, such as communication, self-care, social coexistence and in school activities. Individuals with intellectual disability can and should learn new skills and abilities. However, their development will always be slower when compared to a child with medium intelligence and adaptive competencies [3]. 
Our motivation rises from the desire to provide effective solutions for Web interaction for people with intellectual disabilities. Thus, in this paper, we present a case study on usability evaluation of navigation tasks with this specific group. This case study is divided in two phases: First, we made a Google and SAPO layout comparative study, where the group performed web search tasks using two different search engines; and second, we analyzed two different interaction modalities for inputting textual keywords (through a physical keyboard and a speech recognition application).

In the first phase, we aimed at comparing usability issues of Google and SAPO Web search engines. Knowing that these tools are essential for the retrieval of information in a Web environment [24] and are the first option for people who want to find information [15], it is important to study how people with specific conditions perform basic Web search tasks and, in the end, understand whether they can also use all the advantages that this access can provide.

In the second phase of study, we intended to perform a preliminary evaluation to grasp whether the interaction modalities could interfere with Web interaction in this group of users, and ultimately observe whether they play any role in them having a more autonomous interaction.

For this purpose, we registered and analyzed the gathered data resulting from direct observation, filming, user test evaluation (users' performance, difficulties found, errors, comments/notes) and eye gaze analysis.

At the end of the discussion of the results, we reflect on some perceptions and throw some light on guidelines that we think could help improve usability of search engines, thus allowing an easier and more efficient access for people with intellectual disability.

We consider essential to emphasize the importance of creating new or adapting existing tools for accessing Web content, as it is a basic need, so that discrimination and the digital divide become outdated concepts. Therefore, we think our study proves to be valuable on providing insights on how to bridge the gap in interaction concerning regular users and those with disabilities and to understand what approaches may be taken into account when performing search tasks for this specific group. Also, it can be considered a basis for future research in this area, as it can be extended in various ways: understanding how different search engines may provide better interaction performance; or evaluating more search tasks using other input modalities and devices.

This paper is structured as follows. First, we describe related work. Then, we present the case study conducted: the group of participants involved in the study, the experimental design, the apparatus and the procedure followed. Also, the results and discussion are presented. Then, we present participants' behaviors and difficulties. Also, limitations of this study are noted. Finally, we state the main conclusions of this work and shed some light on possible directions for future research.

\subsection{Related work}

The accessibility of the internet services regarding vulnerable populations, such as individuals with intellectual disabilities, has been studied and widely documented [8, 32, 33]. According to the authors, the use of online services and the internet in general is seen as an opportunity to equally participate in today's information society, largely contributing to the independence and self-determination of these individuals. Although this technology offers great potential, we recognize the fact that the access to these services is still strongly limited concerning the group of people with intellectual disabilities.

One of the characteristics of intellectual disability is cognitive impairment, leading to low levels of literacy. Consequently, users have difficulties in reading and writing. Given that the key basis of a Web search is textual inputs (using keyboards), Web interaction could therefore be compromised.

In fact, due to this situation, several studies have highlighted problems in interaction: Text entry is problematic $[25,26]$, multi-options are referred to as difficult to use [13], as are hyperlinks' recognition [27, 29], typing and reading instructions are difficult to perform [30], knowing whether a word is spelled correctly is complicated [19].

Other studies have even questioned the possibility of finding a guiding principle when planning Web contents (as Web sites) for people with intellectual disability [14] due to difficulties resulting from the wide range of disabilities among this group of people.

An additional problem, sustained by three studies, was that this topic of interest was not considered as important because the researchers' focus shifted and was no longer in the development of Web accessible content, but on the creation of assistive technologies, Web services, browsers or even other technologies such as mobile phones, PDA's, DVD players and cameras [6], despite the interest showed by this group of people in surfing the Web [5, 26].

However, a trend of the studies in this field is the presentation of guidelines and/or recommendations for creating an accessible Web design and/or lists of difficulties in testing [6, 13, 14, 19, 29].

In Friedman and Bryan's [10] study, they conducted a survey of guidelines in the Web design area proposed by different experts and listed these same guidelines. The 22 major recommendations reflect aspects such as size and shape of text, navigation consistency and page design, use of icons, images, written text, style, margins, links, line spacing, and screen layout. They also presented two tables with guidance lists, based on citation frequency. The 
first table refers to the most important recommendations, and the second presents additional recommendations also found by the authors, but that appear less often in the literature $(<15 \%)$.

Widely known and used are the guidelines developed by the World Wide Web Consortium (W3C). There are two versions of these guidelines (WCAG 1.0 and WCAG 2.0). The last version of the guidelines is divided into four global principals thought of to make content visible, operable, understandable and robust. Such principles are based on 12 guidelines that provide the basic goals that web designers and developers should work on to make web content more accessible to users with disabilities. Furthermore, testable criteria success is provided for each guideline to allow WCAG 2.0 to be used to test, for instance, design specification, purchasing, regulation and contractual agreements. There are three levels of conformance: A (lowest), AA, and AAA (highest). These levels were defined to meet the needs of the different groups and situations. A wide variety of techniques for each guidelines and success criteria [31] are also documented. Despite the relevance of these guidelines, they were also object of discussion raising questions whether they are actually sufficient for this specific audience or not [5, 22, 25, 30].

Regarding search engine interaction, Modesto and Ferreira [19] provided guidelines for search features development, by making a comparison between general users and users with low reading skills. As the authors affirm, some of the recommendations presented "were already implemented by Google search engine," but some were new, "for example, presenting less valuable information on demand (as URL), clearly identifying filtered results, providing relevance indications (not only through page ranking), differentiating advertisements from organic results, presenting non-textual media in separate areas and providing tips about how to operate search engine are recommendations that could be explored on this tool" [19].

Another study, carried out by Wong et al. [33], stated that interaction problems are directly related to difficulties regarding basic computer skills, namely interaction with and control of both the mouse and the keyboard, which are linked to the cognitive and sensory-motor functions of individuals [33].

Consequently, it is important more studies focus on users' interaction (in our case, a specific group of users with intellectual disability) specifically related to Web Search engines and to evaluate their experiences [16].

Web search engines are software programs that search documents and files by introducing keywords (user interaction based on understanding/inserting text) and then return a list of documents, by consequence of the entered keywords [18]. A sample list of Web search engines includes: Google, Yahoo!, SAPO, Bing, AltaVista, AOL,
Ask, Medline Plus, Sapo Saúde and Web MD [17, 23]. Of all of these examples, we chose Google and SAPO as the search engines to be studied, mostly because: (1) Google is the most-visited website in the world [1] and one of the most used web search engines [18]; (2) SAPO is a Portuguese Internet service provider that started as a search engine in 1995, and it is the main Portuguese search engine [17] and the sixth-oldest search engine in the world that is still active. This is the fundamental cause for our choice of the search engines in this study.

Aside from understanding the guidelines that can actually influence the user's performance, especially considering people with intellectual disabilities, it has become important to also realize whether the interaction modality itself can improve the interface usability. The goal we strive for today is to be able to give the user the opportunity to focus only on the task at hand, instead of the technology used to interact with the computer/digital platform [7]. Contrarily to the WIMP-based interfaces (Windows, Icons, Menus, Pointing device), natural interfaces permit the user to interact with the interfaces that require a lower cognitive load [4] and thus provide a user-oriented and task-oriented approach [21].

Speech-based user interfaces are considered a solid foundation when handling natural interfaces regarding people with some sort of disability: It permits the input of information without the resort to a keyboard or even in the event of the inexistence of a monitor; it facilitates tasks where hands and/or eyes of the users are busy; and it relieves the need for writing for people with motor or intellectual disabilities [34]. Some systems have been developed that take advantage of speech interfaces in order to replace quick commands given by the mouse or keyboard in graphical interfaces, with very positive results [12, 20].

\section{Case study}

The case study is divided in two different phases: In the first, we intend to compare the usability of the Google versus the SAPO layout; in the second, we expose issues regarding the interaction modalities, using the keyboard and speech-based recognition.

\subsection{Participants}

From thirty-five participants, twenty were invited to partook in the pilot study (eight women and twelve men), whose ages ranged from 19- to 44-year old. These participants were selected by a special education teacher and a psychologist. According to the average rate of literacy and primary education, the participants are comprised between kindergarten to first grade. Seven participants could read and write, while 
the others had great difficulty in these tasks. After 7 years of being part of a previous project of digital integration, we can say that the group presented equal levels of experience in interacting with digital environments, i.e., using the computer with the traditional input devices (mouse and keyboard) and the Internet [28]. Regarding their intellectual disabilities, the individuals were not associated to only one pathology, but a group of pathologies (for example, fetal alcohol syndrome with dysgraphia).

According to DSM-V, these pathologies can be classified according to severity levels, between mild to moderate, but only one of the participants presented a high level of disability [14]. Also, within the group, twelve participants had normal vision; eight had corrected to normal vision. None had motor impairments.

All of the participants were volunteers and had permission of their parents or tutors to perform the tests.

\subsection{Apparatus}

The activities were performed individually, in a controlled environment, with a participant observer (which oriented the user in the task's fulfillment). All tasks were displayed on the computer screen.

The following material resources were used: pen and paper, HP keyboard and optical computer mouse, headphone device with microphone incorporated for audio input, Google's speech input search and an HP computer (AMD Athlon). Note that the user was sitting down at a distance not greater than $1 \mathrm{~m}$ from the computer. Web search engines were displayed on a 15-inch flat-panel monitor, NEC $17^{\prime}$ with a resolution of $1024 \times 768$ pixels. It used the Chrome Web browser, and the Google and SAPO Web search engines. Also, an eye tracking device was used: Tobii Eye-Tracker X50 (Tobii Technology AB, Danderyd, Sweden and Clear View 2.5.1. software), which uses a CCD camera positioned under the computer screen.

\subsection{Experimental design}

The experiment made use of a within-participant repeatedmeasures design. The case study is allied with usability evaluation (user tests). The methods of data collection used are directly related to the research methods adopted.

Before starting the tests, we performed document analysis to study procedures and the experimental design. During the tasks, the direct observation, video recordings and text annotations were very important to register all observation data and oral comments made by the users. Alongside, we performed user tests to register efficiency, effectiveness and satisfaction; in order to overcome some communication difficulties found in the group, we recorded data related to eye gaze with an eye tracking device. Note that this last device is used only for the first phase. After the tests, we performed interviews to better understand users' different attitudes and behaviors throughout interaction; also, we wrote all video and audio annotations in the logbooks to register daily data and evolution on the performance.

Two study phases were prepared for the experiment.

In the first phase, we compared Google and SAPO Web search engines. These two were chosen because they are the two most used tools in the World and in Portugal, respectively, and also because they present two very different layouts.

Google presents a cleaner layout, where there are not many types of contents displayed, only the tools to perform search; as opposed to SAPO, where the tool to perform web search is presented alongside several kinds of other contents: advertising, videos, images and text. With this in mind, we intended to compare the easiness of the navigation tools of the two layouts before the search and also the layout presented in the listing page with the search results.

In the second phase, we proposed search tasks using the keyboard and Google's speech recognition application. Here, we aimed at evaluating two distinct modalities of inputting text. These input modalities were selected because the keyboard is the usual input device for performing Web search tasks, and the Google's speech recognition application could potentially be an alternate option for interaction, as explained in the section about related work. We intended to evaluate the users' performance with these two interaction modalities.

\subsection{Procedures}

Each task was explained before the participant initiated it.

In the first phase of the study, the users were asked to: (1) identify the two search boxes (a key element for accessing information on the Web) on the two Web search engines-locating the text box in order to insert the keyword (search field) and locating the clickable button to proceed with the search (search button) and (2) recognize the results returned by the search in the listing page, also with the two search engines' layouts. In the latter, we observed the easiness of the visual feedback of the listing page and the interpretation of the results obtained. In other words, we examined how the users perceived the search results obtained when they were presented by image or, on the other hand, by text. Therefore, two tasks were defined:

1. They had to perform two Web Searches using the keywords CÃO (portuguese word for dog) and GATO (portuguese word for cat).

2. They had to search for two recipes using the keywords PÃO (portuguese word for bread) and BOLO (portuguese word for cake). 
These tasks were made on both web search engines. In order to ensure randomness, we changed the order of the tasks for each user.

As such, in the first activity, users performed the two tasks previously mentioned twice for each search engine: two searches on SAPO for each of the two tasks and the same on Google. They searched for images of dogs and cats, and recipes for bread and cake. The searches were made in the Portuguese language.

For these tasks, the following steps were followed: First, we chose a participant and explained the intended purpose (step 1); then, with the two search engines open, they were prompted to enter a specific keyword in the search field (step 2). Due to participants' difficulties in reading and writing (specific to their individual disability), keywords were written on a paper and the user had to recognize the characters and replicate them with the keyboard (step 3). Next, the user began the search by clicking in the search icon/text field (step 4). During the task, all observations were recorded. The task ended when the participant clicked on one hyperlink related to the keyword given. Note that: if the keyword was dog or cat, they had to click on an image hyperlink; if it was bread or cake, they had to click on a text hyperlink.

In the second phase, the participants had to perform two search tasks about fruit. Two tasks were also defined:

1. Search for fruit using the keyboard.

2. Search for fruit using speech inputs.

Since in this phase we are dealing with speech-based interfaces, we felt the concern to limit the number of syllables of the spoken words, in order to confer more coherence and consistency in the search for a specific keyword. As such, the words that were chosen had always less than or exactly three syllables.

The steps to successfully complete the tasks were as follows: First, the user chose a fruit to search (step 1); then, with the two search engines opened, we asked the participant to enter the fruit's name in the search field with the keyboard and also with the speech application, spelling the word to the microphone (step 2). All observations were recorded.

The tasks ended when the user clicked on an image hyperlink related to the keyword searched.

Each participant carried out all tasks as previously described.

\section{Results and discussion}

In this section, the results regarding the users' tests (effectiveness, efficiency and satisfaction), the data recorded by eye tracking device, direct observation, video and text annotations are presented and discussed accordingly.

\subsection{Phase 1: usability results regarding Google and SAPO layouts}

In this first phase, we assessed the interface by observing the interaction between a group of people with intellectual disabilities and the layout. For this, we conducted two tasks with Google and two with SAPO. In general, all users liked and appeared excited in performing these tasks.

\subsubsection{Effectiveness results}

Regarding effectiveness, we noted that only one participant did not conclude the tasks. He/she drop out when performing the search on SAPO and, because of that, the participant refused to perform the task on Google. This fact led to the decrease in the success rate in concluding the task on both layouts to $95 \%$.

Also, we registered two specific variables to successfully concluding the task: (1) identification of the search field and (2) identification of the search button.

On the first one, Google registered a success rate of $75 \%$. On the other hand, only $50 \%$ identified the search field using SAPO. Regarding the second variable, identification of the search button, Google presented a success rate of $85 \%$ and SAPO $65 \%$.

Regarding errors, we recorded 125 , being the most observed: wrong character recognition of the keyword and mistakenly entering the keyword with the keyboard.

\subsubsection{Efficiency results}

First, we present the users' average performance time, in seconds, per Web search engine (Fig. 1). Note that in this phase, the input device used was the keyboard and mouse. Also, it is important to notice that the time measured was the time taken to find the search field and button, whereas the time to enter the keyword was not counted.

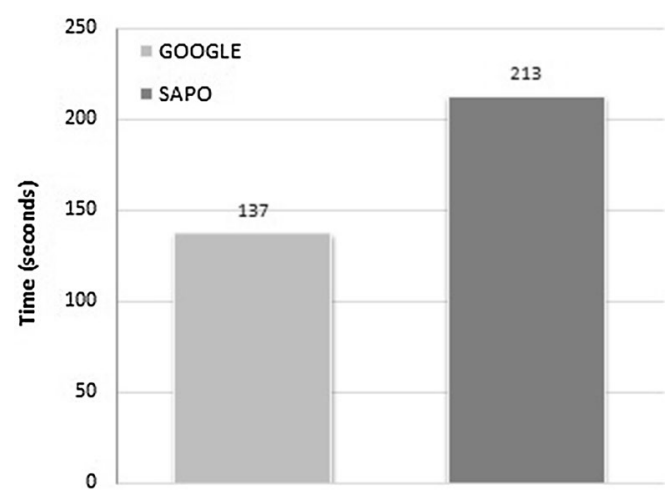

Fig. 1 Users' performance average time (in seconds) per Web search engine 
In Fig. 1, we can see that the users' performance (response time) is better when using the Google Web search engine.

In fact, we can highlight this improvement when analyzing the average time: With Google, users took around 137 s to finish the task; in contrast, with SAPO, they took 213 s. To verify whether this is a significant difference, we applied a $t$ student test, obtaining a level of significance ( $p=0.00)<0.05$, which means that the users' respective performances for the two web search engines are significantly different. These data, along with observations and comments registered in the case study's logbook, reveal that layout complexity is an important variable to analyze. Users actually performed better with a Web search engine that presents a simpler layout. With Google, they rapidly identified the search field and button. With SAPO, users said they did not see where the search boxes were.

Even when we analyzed the average time for each search, we saw similar results (Fig. 2).

Here, we intended to observe whether the user's average performance time would decrease due to the training effect and not the type of keyword.

In Fig. 2, we note that users took less time to finish the tasks on Google, regardless of the order of the performed searches. Also, on this Web search engine, when users performed a second search, the task completion time decreased by $10 \%$. In relation to SAPO, users took more time to finish the tasks. Moreover, when analyzing the order in which the searches were performed, we noted that users took more time to complete the task in the second search: Task completion time increased by $12 \%$.

Likewise, in the observations and comments registered, we noticed that users had less difficulty interacting with Google in the second search. After they understood and had experienced the requested task, their performance was

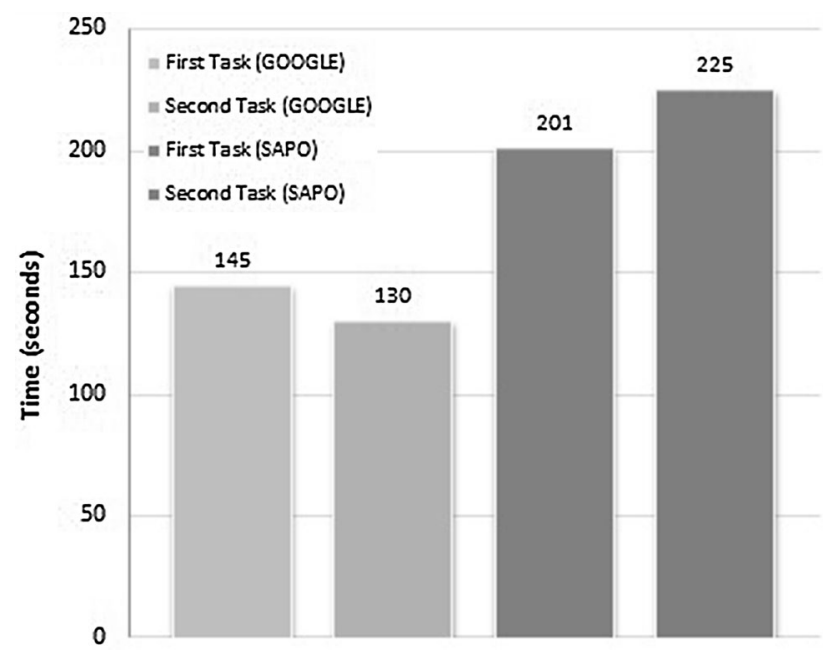

Fig. 2 Users' performance average time (in seconds) per search faster. This appears to indicate that apprenticeship helps them with the task comprehension and consequently the interaction with this search engine.

On the other hand, with SAPO, despite the apprenticeship experience with the first search, users did not improve their performance and the task completion time increased, as previously seen. Users seemed to be lost and even commented that they were unable to find the tools to repeat the search (search field and button) because they did not know where they were located and kept asking: "Where is it?" They seemed confused with the large flow of information (so many contents) presented by this Web search engine.

Also, we highlight some important user behaviors:

One user did not perform one of the tasks on Google (search for a cat image). He started one task on SAPO (search for a dog image) and because he found the task very difficult to complete; he refused to finish the activity or carry out the other tasks on Google. It was very hard to find the button and search field, so he became frustrated with the complexity of the task. The user tried the search engine once and then formed an (emotional) opinion about not only the task, but the overall activity. As a result, the user was not satisfied with the interaction and eventually quit.

Secondly, we noticed that users who performed well on SAPO were those who had an improved ability to read and write, rather than those who had more experience with technology (Internet, computer and input devices), despite also having these abilities. We think this happened because, despite all the information presented, they did not feel lost and rapidly managed to find the search field using the scroll.

Finally, the observations recorded helped us determine that the layout design can influence interaction: Users felt lost when presented with a lot of information. This also made them frustrated because they could not identify the search button or search field, making them quit tasks and raising fears of trying again in another session.

Next, Fig. 3 shows major difficulties observed.

The difficulties experienced by most users were related to the identification of the research field, identification of the search button, clicking on the search field to start writing the keyword and whether the user identified the image or text hyperlink requested at the beginning of the task.

In the first search of task 1 (search for a dog image on both Web search engines), all users identified the image of a dog on both Web engines. For the other variables, they showed more difficulties when using SAPO. Specifically, five users had difficulties identifying the search field; four had difficulties identifying the search button; and six had difficulties clicking on the search field to start writing. 


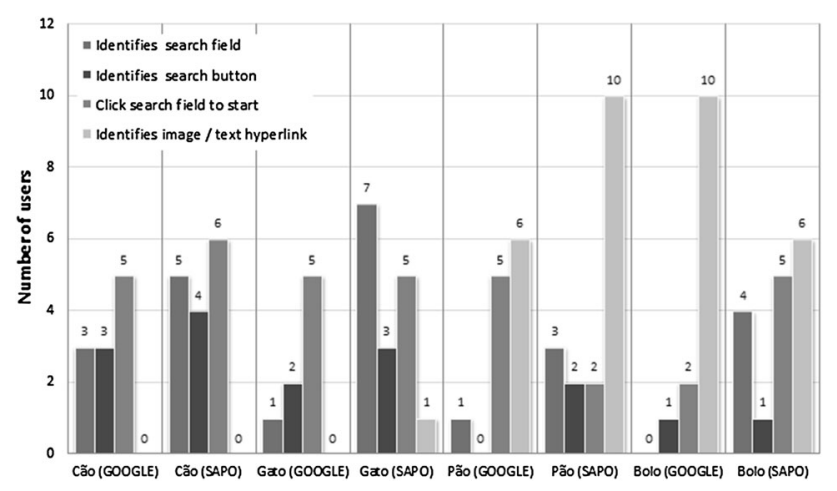

Fig. 3 Comparison of types of difficulties observed per task

On Google, the major difficulty was in perceiving the need to click on the search field to start writing the keyword (five users). Only three users had problems with the other two variables.

In the second search (search a cat image on both Web search engines), again more difficulties were recorded on SAPO: Seven users did not identify the search field; three had trouble finding the search button; five did not click on the search field to start writing; and one person had difficulties clicking on the cat image as requested. On the other hand, on Google, all the users were able to find and click on the cat image. The major difficulty was clicking on the search field to start writing (five users), while only two users had difficulties in finding the search button, and one had trouble finding the search field.

In the first search of task 2 (search a bread recipe on both Web search engines), on SAPO, three users did not find the search field and two did not find the search button. Again, two did not click on the search field to start writing, and ten had difficulties in finding the text hyperlink requested at the beginning. On Google, only one user had trouble finding the search field, but everybody was able to identify the search button. Five users still had difficulties clicking on the search field to start writing, and six could not find the text hyperlink without help. Here, the search button was represented by an image (magnifying glass); on the other hand, on SAPO, this button was represented by text (with the word Search in Portuguese). This could be the differentiation factor. They could rapidly identify the Google icon search button but took more time to identify the SAPO text search button.

In the second search of this task (search for a cake recipe on both Web search engines) on SAPO, four users did not find the search field, one did not find the search button, five had difficulties clicking on the search field to start writing, and six had difficulties finding the text hyperlink requested at the beginning. On Google, all users found the search field, and only one user had troubles identifying the search button; two had difficulties on clicking the search field to start writing, and ten users did not find the text hyperlink without help.

We noticed that when the results were presented in text, users had much more difficulties in successfully completing the task. Users presented low literacy levels and many difficulties in interpreting/decoding text so they were able to identify images much more quickly and preferred clicking on them. They paid less attention to text contents.

Another important observation was that after users finished the task (find and click the image or text hyperlink requested), they continued searching (even when the task was terminated). They did this by clicking surrounding links, which were near to the requested one, i.e., they did not return to the search field to conduct a new search (this only happened when requested).

\subsubsection{Eye tracking results}

In addition, the results of the images obtained using an eye tracking device are presented. More precisely, images of hotspots (areas where the user focuses attention) and also gazeplots (the visual path taken by users) are presented to identify whether the users recognized the search field and search button. In Fig. 4, the individual hotspots of the group with intellectual disability are shown for Google (Fig. 4).

The highest degree of this groups' attention was on the search field and search button on Google's search engine. When such focus did not occur, the degree of attention was focused very close to the field and button. The focus on the surrounding area may have occurred because some individuals had difficulties looking straight forward (characteristic of the disability), so their focus of attention as marked by the eye tracking devices differed. This is more precisely shown in the gazeplots presented in Fig. 5.

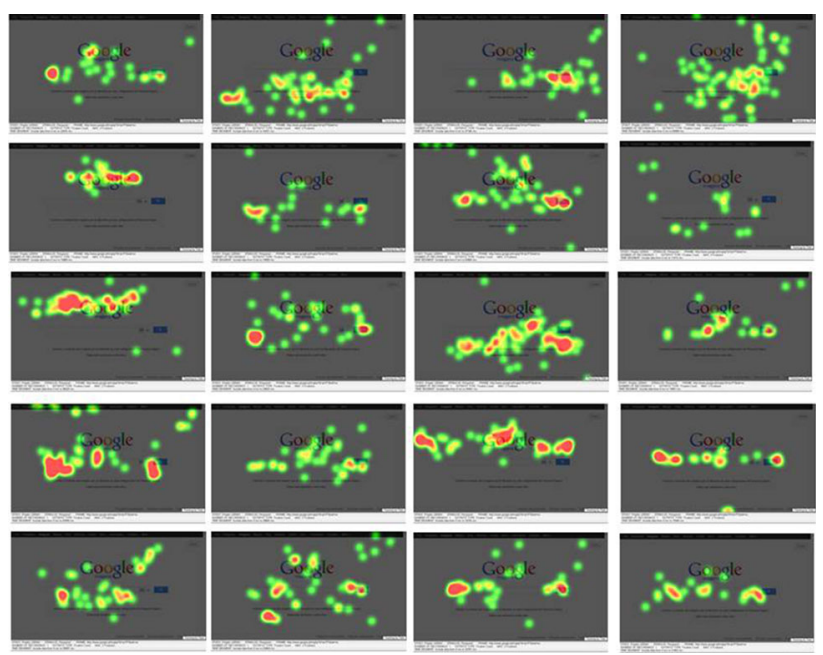

Fig. 4 Eye tracking images (individual hotspots on Google platform) 


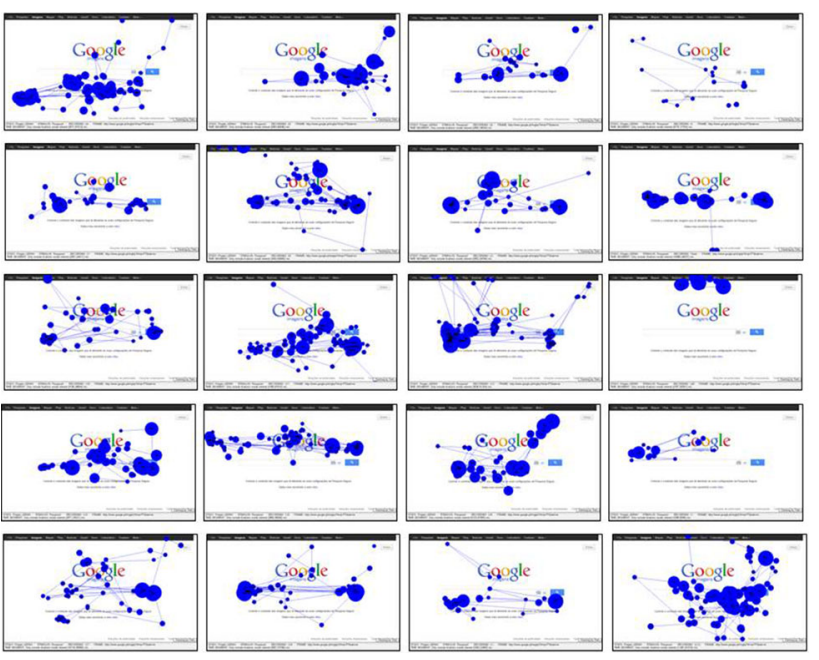

Fig. 5 Eye tracking images (individual gazeplots on Google platform)

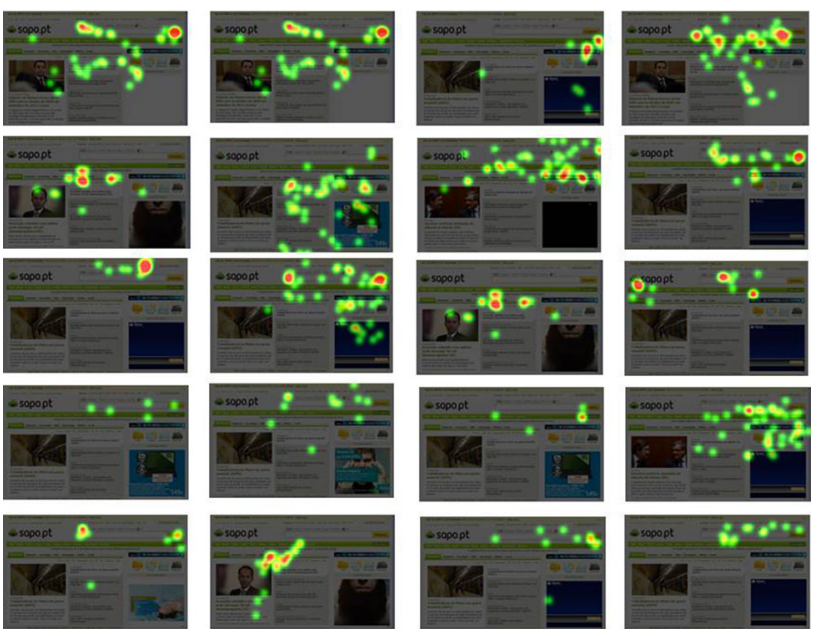

Fig. 6 Eye tracking images (individual hotspots on SAPO platform)

Here, the visual focus concentration on the search field and button became more obvious. In most individual gazeplots shown this concentration, almost all users identified the search field and button (gazeplots are registered above the field). As previously mentioned, it is once again visible, in at least two gazeplots, that the vision path is above the Google' logo, and there is no registry on the search field or button. This happens because some users had difficulty looking forward due to their disability. Note that all tasks presented were performed successfully, regardless of having a proper vision registry (above search field and button). Figure 6 presents the SAPO eye tracking device results.

On SAPO (Fig. 7), a major level of vision focus dispersion is recorded on the background. This is easy to observe in SAPO's gazeplot results (Fig. 7)
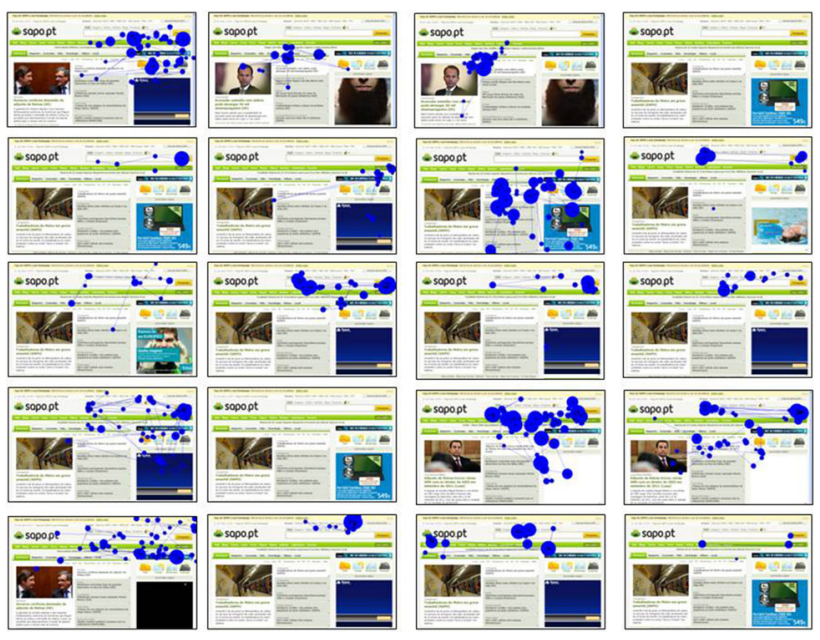

Fig. 7 Eye tracking images (individual gazeplots on SAPO platform)

On SAPO's gazeplots, the dispersion focuses on all the content presented in the background area. Here, we had five cases where there is no registry above the search field or button for the vision path; in fact, two gazeplots have no path at all.

When comparing the results of the two layouts and the observations/comments registered in the case study's logbook, it appears that users memorize the search field's design and then look for it on the layout. All the content displayed on SAPO (news, advertising, search field, buttons, hyperlinks) confuses the user-especially videos that start automatically - consequently frustrating him or her. In those cases, the users felt lost and asked for help to complete the task at hand.

This seems to indicate that complexity of layout is an important variable for a good user-Web interaction because if the layout does not present large amounts of information (cleaner design), users with intellectual disability can more easily find the search button and field (important steps to perform a Web search).

Another observation mentioned above is that users seem to clearly identify image contents (as the Google' icon search button) and have more trouble identifying SAPO's text search button.

\subsection{Phase 2: usability results regarding interaction modalities}

An additional issue that was one of the major difficulties detected was entering the keyword. Searching by entering characters is the most usual method of web search. There are hundreds of search engines which use text and keywords as a search method. However, users encounter difficulties when inserting keywords without help and interacting with the keyboard. Thus, we see the great need 
to register these data as well, because if users cannot handle the keyboard and insert keywords, the execution of search tasks on the Web can be compromised. So it is important to analyze how users perform their interaction with the usual input devices (keyboard and mouse) and provide an alternate interaction mode to help overcome possible problems that arise from these difficulties. Eventually, in order to help users understand and perform search tasks online, they could perhaps be given an optional tool for interaction that could provide autonomy.

With this in mind, we decided to make this preliminary study to understand whether this interaction mode could have potential for this group of users and improve their performance.

The alternative interaction mode used in this study was a speech-based interface. This is a field that is not widely explored for this group of users [2]. Google has added a tool in the Chrome browser that consists on the introduction of keywords through speech. This feature can combine words into various search terms, and the use of bigger keywords could enlarge the search. The difference between this and other speech applications is that it does not require a training phase for subsequent speech recognition and can be used on any Web site that has a search field. Even though this application does not require a specific calibration, we chose to use because it is free and publicly accessible.

Regarding the interaction with the keyboard and speechbased application, we present users' global performance time per interaction mode (Fig. 8).

In Fig. 8, we note that only five users (25\%) managed to complete the task successfully with the speech-based application, while all were able to finish the task using the keyboard.

However, we note that the interaction with the keyboard was never made autonomously, as all the users needed help in writing the keyword on a paper in order to replicate it with the keyboard.

When we compared user's successful task results with the two interaction modes (using keyboard and speech-

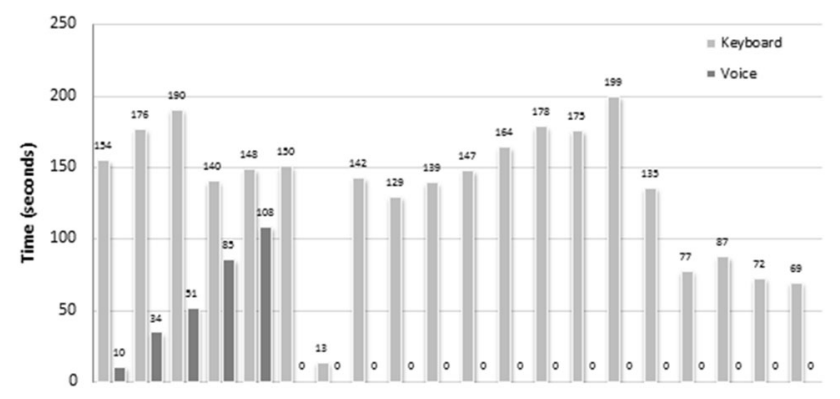

Fig. 8 Users' performance average time per interaction mode (keyborad and voice) based application), we observed that users performed better using the Google speech-based application. The major problem observed during the test was the speech recognition itself. It had nothing to do with users' disability; rather, it focused on word pronunciation. It is likely that even users without disabilities could perform this task unsuccessfully due to poor word pronunciation. We think that if this problem could be corrected, users would be able to perform Web search tasks using speech recognition interfaces without help, i.e., autonomously.

In particular, the difficulties observed in completing these tasks were: for the keyboard, character recognition and reproducing; for the speech-based application, correct keyword pronunciation.

\section{Participants' behaviors and difficulties in web interaction: proposed recommendations}

During this study, we observed some users' behaviors and difficulties caused by the layout design, directly influencing their interaction. Thus, we felt important to report these difficulties by presenting them as recommendations to follow as good practice. Also, we compared them with other guidelines from previous studies.

First, we observed that when the search engine layout presented different contents (as image, video, audio and text), in various formats (such as news, advertising, search fields or merely buttons or hyperlinks), users felt lost and asked for help to finish the task as the layout presented was complex and overwhelmed with information. Often they comment: "where is this...?" or "where am I?" So, our first recommendation is that search engines layouts should be simple.

The first principle of the WCAG 2.0 (Perceivable) states that "information and user interface components must be presentable to users in ways they can perceive" and makes explicit, in the guideline 1.3, that developers must "create content that can be presented in different ways (for example, simpler layout) without losing information or structure." Here, a cleaner layout is also presented as an option to help users in such a way that they could understand and interact with the digital content.

Similarly, Modesto and Ferreira [19] regarding the creation and improving of search tools recommend that developers and designers minimize distractions: The focus must be in the task to be performed and one activity at a time [19].

Second, we noticed users memorized the search field and button's design and location on the page, and then, they looked for it on the layout. Again showing that complexity of the layout is an important variable: If the layout does not present a lot of information (i.e., has a 
simpler design), the search button and field are easier to find. This is also visible in the users' successful results with Google's layout. Thus, we support the recommendation: Search button and field should appear in a prominent place for easy identification.

In the work of Friedman and Bryen [10], a list of design recommendations is presented, based on citation frequency, for users with cognitive disabilities. Here, we can find the recommendation "Navigation buttons clear, large and consistent" is frequently cited, i.e., $20 \%$ of the studies acknowledge the importance of these buttons and their clear, large and consistent presentation for an easy recognition [10].

Third, users seemed to be confused with contents that start automatically, such as advertisements/pop-ups. These kinds of contents should not be presented in the layout (in order to make sure that layout is as simple as possible), but if they are included, they should not start automatically. The recommendation presented here is: Multimedia contents/pop-ups should not start automatically.

Modesto and Ferreira [19] reiterated the importance of the media only being presented upon user request and also added that these results must be presented in a separate area of the page. Friedman and Bryen [10] encountered $10 \%$ of citation frequency that recommended no time-based events.

Forth, regarding search results, we noted users don't pay attention to text, due to low literacy levels and the many difficulties in the interpretation/decoding of text. In this regard, it seems that other kinds of content needs to be presented to increase attention and interest in the task/activity. This occurred when the results were presented with images. The recommendation here is: Search results should be presented in images.

The importance of images, for users with intellectual disabilities is supported in many studies. Friedman and Bryen [10] counted $75 \%$ of frequency citing the guideline that recommends images along with text. Rocha [28] embraced the possibility of designing accessible icons, fully recognized by users with intellectual disability, and developed different studies to support the design model to create icons accessible for this group of people [28].

Fifth, along with the recommendation previously defined and reaffirming the importance of images, we registered that users remembered and preferred the Google search button (that uses a magnifying glass icon) instead of the SAPO search button (that uses text). So the recommendation proposed is: Use buttons with images instead of text.

Finally, concerning the speech-based interface, Google's speech application appears to be ineffective because of the speech recognition issues (incorrect pronunciation of keywords). However, other applications may perform better and avoid these detected flaws. We think this could be a good interaction modality for future work, maybe using other more effective recognition softwares. Thus, the recommendation proposed here is: Speech recognition for search could be an alternative for interaction.

Indeed, it enables the input of information without the resort to a keyboard or even when a monitor is unavailable, relieving the need for written commands for people with disabilities or motor impairments [34]. In their work, Davies et al. [8] also indicated an addition of a text-tospeech technology to the specific browser they presented, aiming to enhance independent use by individuals with mental retardation [8].

\section{Limitations of the study}

One limitation of the study is that we do not use a control group (with or without disabilities) to compare usability results. Also, we feel that a usability analysis is not enough on its own, and thus, we will proceed with a Fitts' law analysis for a comprehensive study of the performance/ accuracy regarding different tasks' levels of difficulty.

Furthermore, we intend to increase the number of participants and perform more activities relating to elemental tasks, such as selection, insertion and manipulation.

Concerning the speech-based application, although we consider an appropriate choice since it was a free and opensource software, there were some limitations inherent of the application: No need for speech calibration stood as an obstacle to the reliability of the interaction. In addition, the incorrect pronunciation of the keyword was another limitation to the successful completion of tasks.

\section{Conclusions}

In this study, we reveal how a group of people with intellectual disability performs a Web search task using two different input modalities. This interaction was assessed by using two different Web engine search browsers (Google and SAPO).

After the results obtained, we observed that the Google search engine is more easy to use, effective and efficient for users with intellectual disability than SAPO. Globally, users were faster, made less errors, experienced less difficulties and were more satisfied with Google than SAPO. For this group of users, regardless of the size of the keyword or the order of the search, Google was the search engine where they presented a better performance.

Likewise, we concluded with the direct observations and comments registered in the logbooks that layout complexity (assessed by the amount of information displayed 
on the home page) is an important variable in user interaction with Web search engines.

Concerning the interaction with the input devices, we noticed that when using a keyboard, participants were capable of inputting text. However, this type of input does not give them autonomy, because they needed help in understanding which keyword to search for: First, the keyword was written on a paper so that users could replicate it on the keyboard. Without this step, users could not proceed with the task. The Google speech-based application removed this need for interpreting the text but had problems with the actual keyword pronunciation.

Once again, we also highlight these users' satisfaction when interacting with the digital world.

It was important to study how a group of people with intellectual disabilities perform basic Web search tasks to endorse recommendations for enhancing accessibility of digital environments and ultimately provide them with a more autonomous interaction.

\section{Future work}

In the near future, we will test other Web search engines in order to replicate these results and look at other input devices for user-Web interaction, such as touch in mobile devices (tablets and smartphones) and touch screens, aiming to assess the usability of these tools. We intend to determine whether these can be accessible alternatives. We will also study how we can create accessible images/hyperlinks to substitute text hyperlinks. In the end, everything possible must be done to give people with intellectual disabilities autonomy in performing Web search tasks. We also intend to conduct a more thorough study on the input modalities (keyboard and speech-based application), and we will also consider other natural interfaces.

\section{References}

1. Alexa: Top 500 global sites http://www.alexa.com/topsites/ (2010)

2. Aylett, M.P., Kristensson, P.O., Whittaker, S., Vazquez-Alvarez, Y.: None of a CHInd: relationship counselling for HCI and speech technology. In: Proceeding CHI'14 Extended Abstracts on Human Factors in Computing Systems, ACM New York, NY, USA, pp. 749-760, 2014. ISBN: 978-1-4503-2474-8. doi:10. 1145/2559206.2578868. http://dl.acm.org/citation.cfm?id=25592 06.2578868

3. American Psychological Association (APA): DSM-V-The Diagnostic and Statistical Manual of Mental Disorders, vol. 5. http:// www.dsm5.org/Pages/Default.aspx (2013)

4. Blake, J.: The natural user interface revolution. In: Natural User Interfaces in .NET, 15th edn., pp. 1-43. Manning Publications (2012)
5. Bohman, P.R.: Functional cognitive disabilities. http://paulboh man.com/accessibility/cognitive/functional (2007)

6. Carey, C.C., Friedman, M.G., Bryen, D.N.: Use of electronic technologies by people with intellectual disabilities. Ment. Retard. 43(5), 322-333 (2005)

7. Dam, A.V.: Post-WIMP user interfaces. Commun. ACM 40(2), 63-67 (1997)

8. Davies, D.K., Stock, S.E., Wehmeyer, M.L.: Enhancing independent internet access for individuals with mental retardation through use of a specialized web browser: a pilot study. Educ. Train. Ment. Retard. Dev. Disabil. 1(36), 107-113 (2001)

9. Dewan, S., Ganley, D., Kraemer, K.L.: Across the digital divide: a cross-country multi-technology analysis of the determinants of IT penetration. J. Assoc. Inf. Syst. 6(12), 409-432 (2005)

10. Friedman, M., Bryen, D.: Web accessibility design recommendations for people with cognitive disabilities. Technol. Disabil. 9, 205-212 (2007). ISSN 1055-4181/07/\$17.00

11. Godinho, F.: Uma nova abordagem para a formação em Engenharia de Reabilitação em Portugal, Ph.D. Thesis, University of Trás-os-Montes e Alto Douro (2010). http://repositorio.utad.pt/ bitstream/10348/572/1/Phd_fafbgodinho.pdf

12. Gorniak, P., Roy, D.: Augmenting user interfaces with adaptive speech commands. In: Proceedings of the 5th international conference on Multimodal interfaces-ICMI'03, New York, New York, USA, p. 176 (2003)

13. Harrysson, B.: Internet Enabling Design for People with Cognitive Limitations. Lund University, Sweden (2003)

14. Harrysson, B., Svensk, A., Johansson, G.: How people with developmental disabilities navigate the internet. Br. J. Spec. Educ. 30(3), 138-142 (2004)

15. Kim, J.: Describing and predicting information-seeking behavior on the Web. J. Am. Soc. Inf. Sci. Technol. 60(4), 679-693 (2009)

16. Leroy, G., Xu, J., Chung, W., Eggers, S., Chen, H.: An end user evaluation of query formulation and results review tools in three medical meta-search engines. Int. J. Med. Inform. 76, 780-789 (2007). http://dx.doi.org/10.1016/j.ijmedinf.2006.08.001

17. Lopes, C. T., Ribeiro, C.: Comparative evaluation of web search engines in health information retrieval. Online Inf. Rev. 35(60), 869-892 (2011). http://dx.doi.org/10.1108/14684521111193175

18. Malik, S.: A comparative study of two major search engines: Google and Yahoo. Orient. J. Comp. Sci. Technol, 1(7) (2014). http://computerscijournal.org/?p=706

19. Modesto, D.M., Ferreira, S.B.L.: Guidelines for search features development-a comparison between general users and users with low reading skills. In: 5th International Conference on Software Development and Technologies for Enhancing Accessibility and Fighting Info-exclusion, DSAI 2013, vol. 27, pp. 334-342. Elsevier. Procedia Computer Science (2013)

20. Nakano, T.: Flexible shortcuts. In: Proceeding of the TwentySixth Annual CHI Conference Extended Abstracts on Human Factors in Computing Systems-CHI'08, New York, New York, USA, p. 2621 (2008)

21. Nielsen, J.: Noncommand user interfaces. Commun. ACM 36(4), 83-99 (1993)

22. Opitz, C.: The effects of implementing web accessibility standards on the success of secondary adolescents with learning disabilities. Ph.D. Thesis, Arizona State University (2003). http:// worldcat.org/oclc/55135520

23. Palanisamy, R.: Evaluation of search engines: a conceptual model and research issues. Int. J. Bus. Manag. Can. Cent. Sci. Educ. 8(6) (2013) ISSN 1833-3850 E-ISSN 1833-8119. http://www.ccsenet. org/journal/index.php/ijbm/article/view/20708

24. Rangaswamy, A., Giles, C. L., Seres, S.: A strategic perspective on search engines: thought candies for practitioners and researchers. J. Interact. Mark. (23), 49-60 (2009). http://dx.doi. org/10.1016/j.intmar.2008.10.006 
25. Rocha, T.: Acessibilidade e Usabilidade na Internet para Pessoas com Deficiência Intelectual. M.Sc. Thesis, University of Trás-osMontes e Alto Douro (2008)

26. Rocha, T., Gonçalves, M., Godinho, F., Magalhães, L., Bessa, M.: Accessibility and usability in the internet for people with intellectual disabilities. In: Proceedings of 2nd International Conference on Software Development for Enhancing Accessibility and Fighting Info-exclusion. DSAI'2009, Lisboa, pp. 25-29 (2009)

27. Rocha, T., Bessa, M., Gonçalves, M., Cabral, L., Godinho, F., Peres, E., Reis, M., Magalhães, L., Chalmers, A.: The recognition of web pages' hyperlinks by people with intellectual disabilities: an evaluation study. J. Appl. Res. Intell. Disabil. 25(6), 542-552 (2012). doi:10.1111/j.1468-3148.2012.00700.x

28. Rocha, T.: Metáfora de Interação para o Acesso à Informação Digital de uma Forma Autónoma por Pessoas com Deficiência Intelectual. Ph.D. Thesis, University of Trás-os-Montes e Alto Douro (2014)

29. Roh, S.Z.: Designing accessible Web-based instruction for all learners: perspectives of students with disabilities and Web-based instructional personnel in higher education. Unpublished
Doctoral dissertation, Indiana University (2004). http://mutex. gmu.edu:2068/pqdweb?did=828407271\&sid=3\&Fmt=2\&clientId= $31810 \& R Q T=09 \&$ VName $=P Q D$

30. Small, J., Schallau, P., Brown, K., Ettinger, D., Blanchard, S., Krahn, G.: Web accessibility for people with cognitive disabilities. http://www.resna.org/ProfResources/Publications/Proceed ings/2005/Research/CAC/Small.php (2005)

31. W3C, World Wide Web Consortium-WAI, Web Accessibility Initiative: Web Content Accessibility Guidelines (WCAG) 2.0. http://www.w3.org/TR/WCAG20/ (2008)

32. Williamson, K., Schauder, D., Stockfield, L., Wright, S., Bow, A.: The role of the internet for people with disabilities: issues of access and equity for public libraries. Aust. Libr. J. 50(2), 157-174 (2001). doi:10.1080/00049670.2001.1075595

33. Wong, A.W.K., Chan, C.C.H., Li-Tsang, C.W.P., Lam, C.S.: Competence of people with intellectual disabilities on using human-computer interface. Res. Dev. Disabil. 30, 107-123. Elsevier (2009). http://www.sciencedirect.com

34. Yankelovich, N., Lai, J.: Designing speech user interfaces. In: CHI'99 Extended Abstracts on Human Factors in Computing Systems-CHI'99, New York, New York, USA, p. 124 (1999) 